\title{
Phase Equilibria of Quasi-Ternary Systems Consisting of Multi-Component Polymers in a Binary Solvent Mixture VI. Theoretical Prediction of the Flory Solvent Composition
}

\author{
Shigenobu MATSUDA and Kenji KAMIDE* \\ Fundamental Research Laboratory of Fibers and Fiber-Forming Polymers, \\ Asahi Chemical Industry Co., Ltd., \\ 11-7 Hacchonawate, Takatsuki, Osaka 569, Japan \\ (Received May 15, 1986)
}

\begin{abstract}
An attempt was made to present a theoretical background for calculating the Flory (theta) solvent composition of quasi-ternary systems consisting of multicomponent polymers dissolved in a binary solvent mixture. For this purpose, Kamide and Matsuda's theory on the critical point of the quasi-ternary system [Polym. J., 18, 347 (1986)] was used as a basic principle. The volume fractions of solvents 1 and 2 at Flory solvent conditions $v_{1}{ }^{\mathrm{F}}$ and $v_{2}{ }^{\mathrm{F}}$ can be regarded as those at the critical solution points $v_{1}{ }^{\mathrm{c}}$ and $v_{2}{ }^{\mathrm{c}}$ at limit of an infinite molecular weight and the following equation was derived:

$$
\left(\frac{1}{v_{1}{ }^{\mathrm{F}}}+\frac{1}{v_{2} \mathrm{~F}}-2 \chi_{12}\right)\left(\frac{1}{v_{1} \mathrm{~F}}-2 \chi_{13}\right)-\left(\frac{1}{v_{1} \mathrm{~F}}+\chi_{23}-\chi_{13}-\chi_{12}\right)^{2}=0
$$

where $\chi_{12}, \chi_{13}$, and $\chi_{23}$ are the thermodynamic interaction parameters between the two components chosen among solvents 1,2 , and the polymer (3). By solving the equation $v_{1}{ }^{\mathrm{F}}\left(=1-v_{2}{ }^{\mathrm{F}}\right)$ can be theoretically calculated from knowledge on $\chi_{12}, \chi_{13}$, and $\chi_{23}$. Effects of $\chi_{12}, \chi_{13}$, and $\chi_{23}$ on $v_{1}{ }^{\mathrm{F}}$ were investigated systematically with computer experiments. It is predicted that double Flory solvent compositions have a possibility to exist for some polymer-solvents system. When $\chi_{12}>0$, mixing of two good solvents is expected to yield a Flory solvent mixture.
\end{abstract}

KEY WORDS Quasi-Ternary System / Flory (Theta) Solvent / Thermodynamic Interaction Parameter / Computer Experiment / Critical Solution Point /

The Flory solvent composition or the Flory temperature of binary solvent mixture consisting of solvent 1 and solvent 2 for a given polymer is usually experimentally determined ${ }^{1}$ by (1) the second virial coefficient $A_{2}$ in light scattering, membrane osmometry or sedimentation equilibrium, ${ }^{2,3}$ (2) cloud point ${ }^{\circ}$ titration, ${ }^{4-6}$ (3) phase equilibrium ${ }^{1,2,7-9}$ (4) the exponent of the Mark-Houwink-Sakurada equation, ${ }^{2,3,10}$ and (5) concentration dependence of hydrodynamic parameters such as sedimentation and diffusion coefficients. ${ }^{11}$

The article attempts to present an alternative theoretical method for evaluating the Flory solvent composition (i.e., the volume fraction of the solvent $1 v_{1}{ }^{\mathrm{F}}$ and that of the solvent $2 v_{2}{ }^{\mathrm{F}}$ ) at a given temperature from three thermodynamic interaction parameters $\chi\left(\chi_{12}, \chi_{13}\right.$, and $\left.\chi_{23}\right)$ (Here, suffix 1,2 , and 3 denote solvent 1 , solvent 2 and the polymer, respectively).

\footnotetext{
* To whom all correspondences should be addressed.
} 


\section{THEORETICAL BACKGROUND}

Very recently, we showed for multicomponent polymers/solvent 1 /solvent 2 system that the spinodal and the neutral equilibrium conditions are expressed by ${ }^{12}$

$$
\begin{aligned}
& \left(\frac{1}{v_{1}}+\frac{1}{v_{2}}-2 \chi_{12}\right)\left(\frac{1}{v_{\mathrm{p}} X_{w}{ }^{0}}+\frac{1}{v_{1}}-2 \chi_{13}\right) \\
& -\left(\frac{1}{v_{1}}+\chi_{23}-\chi_{13}-\chi_{12}\right)^{2}=0 \\
& {\left[\frac{1}{v_{\mathrm{p}} X_{w}{ }^{0}}\left(\frac{1}{v_{1}{ }^{2}}-\frac{1}{v_{2}{ }^{2}}\right)+\frac{1}{v_{1}{ }^{2}}\left(\frac{1}{v_{2}}-2 \chi_{23}\right)\right.} \\
& \left.-\frac{1}{v_{2}{ }^{2}}\left(\frac{1}{v_{1}}-2 \chi_{13}\right)\right]\left(\frac{1}{v_{\mathrm{p}} X_{w}{ }^{0}}+\frac{1}{v_{1}}-2 \chi_{13}\right) \\
& -\left(\frac{1}{v_{1}}+\chi_{23}-\chi_{13}-\chi_{12}\right)\left[\frac{1}{v_{1}{ }^{2}}\left(\frac{1}{v_{2}}-2 \chi_{23}\right)\right. \\
& +\frac{1}{v_{1}{ }^{2} v_{\mathrm{p}} X_{w}{ }^{0}}+\frac{X_{z}{ }^{0}}{v_{p} X_{w}{ }^{0}}+\left\{\frac{1}{v_{1} v_{2}}-\frac{2 \chi_{13}}{v_{2}}-\frac{2 \chi_{23}}{v_{1}}\right. \\
& +2\left(\chi_{12} \chi_{13}+\chi_{13} \chi_{23}+\chi_{23} \chi_{12}\right) \\
& \left.\left.-\left(\chi_{12}{ }^{2}+\chi_{13}{ }^{2}+\chi_{23}{ }^{2}\right)\right\}\right]=0
\end{aligned}
$$

in

\section{(v)} ly, $1 /\left(v_{\mathrm{p}}{ }^{\mathrm{c}} X_{w}{ }^{0}\right)$ is of order of $\left(X_{w}{ }^{0}\right)^{a-1}($ i.e., $1 /$
$\left(v_{\mathrm{p}}{ }^{\mathrm{c}} X_{w}{ }^{0}\right) \sim O\left(\left(X_{w}{ }^{0}\right)^{a-1}\right)$ and at limit of $X_{w}{ }^{0} \rightarrow$
$\infty 1 /\left(v_{\mathrm{p}}{ }^{\mathrm{c}} X_{w}{ }^{0}\right)$ approaches to zero.

Equation 2 can be rewritten by dividing both sides by $X_{z}^{0}$ as

$$
\begin{aligned}
{\left[\frac{1}{v_{\mathrm{p}} X_{w}{ }^{0}\left(X_{z}{ }^{0}\right)^{1 / 2}}\left(\frac{1}{v_{1}{ }^{2}}-\frac{1}{v_{2}{ }^{2}}\right)+\frac{1}{v_{1}{ }^{2}}\left(\frac{1}{v_{2}}-2 \chi_{23}\right)\right.} \\
\left.\times \frac{1}{\left(X_{z}^{0}\right)^{1 / 2}}-\frac{1}{v_{2}{ }^{2}}\left(\frac{1}{v_{1}}-2 \chi_{13}\right) \frac{1}{\left(X_{z}^{0}\right)^{1 / 2}}\right] \\
\times\left[\frac{1}{v_{\mathrm{p}} X_{w}{ }^{0}\left(X_{z}^{0}\right)^{1 / 2}}+\frac{1}{v_{1}\left(X_{z}{ }^{0}\right)^{1 / 2}}\right. \\
\left.-\frac{2 \chi_{13}}{\left(X_{z}{ }^{0}\right)^{1 / 2}}\right]-\left(\frac{1}{v_{1}}+\chi_{23}-\chi_{13}-\chi_{12}\right) \\
\times\left[\frac{1}{v_{1}{ }^{2}}\left(\frac{1}{v_{2}}-2 \chi_{23}\right) \frac{1}{X_{z}{ }^{0}}+\frac{1}{v_{1}{ }^{2} v_{\mathrm{p}} X_{w}{ }^{0} X_{z}{ }^{0}}\right. \\
+\frac{1}{v_{\mathrm{p}} X_{w}{ }^{0}}\left\{\frac{1}{v_{1} v_{2}}-\frac{2 \chi_{13}}{v_{2}}-\frac{2 \chi_{23}}{v_{1}}\right. \\
+2\left(\chi_{12} \chi_{13}+\chi_{13} \chi_{23}+\chi_{23} \chi_{12}\right) \\
\left.\left.-\left(\chi_{12}{ }^{2}+\chi_{13}{ }^{2}+\chi_{23}{ }^{2}\right)\right\}\right]=0
\end{aligned}
$$

The left hand side of eq 5 reduces to zero at limit of $X_{w}{ }^{0} \rightarrow \infty$ regardless of $v_{1}$ and $v_{2}$. Therefore, $v_{1}{ }^{\mathrm{c}}$ and $v_{2}{ }^{\mathrm{c}}$ at limit of infinite $X_{w}{ }^{0}$ (in other words, $v_{1}{ }^{\mathrm{F}}$ and $v_{2}{ }^{\mathrm{F}}$ ) can be calculated by solving eq 4 alone for a given combination of $\chi_{12}, \chi_{13}$, and $\chi_{23}$ under the condition that $0<v_{1}<1$ and $0<v_{2}<1$.

\section{COMPUTER EXPERIMENT}

Replace $v_{1}$ and $v_{2}$ in eq 4 with $v_{1}{ }^{\mathrm{F}}$ and $1-v_{1}{ }^{\mathrm{F}}$ 
$\left(v_{2}{ }^{\mathrm{F}}\right)$, respectively and denote the left-hand side term of the equation by $A$ in the following manner:

$$
\begin{aligned}
A \equiv & \left(\frac{1}{v_{1}^{\mathrm{F}}}+\frac{1}{1-v_{1}{ }^{\mathrm{F}}}-2 \chi_{12}\right)\left(\frac{1}{v_{1}^{\mathrm{F}}}-2 \chi_{13}\right) \\
& -\left(\frac{1}{v_{1}{ }^{\mathrm{F}}}+\chi_{23}-\chi_{13}-\chi_{12}\right)^{2}=0
\end{aligned}
$$

For a given combination of $\chi_{12}, \chi_{13}$, and $\chi_{23}$, $v_{1}{ }^{\mathrm{F}}$ (and accordingly, $v_{2}{ }^{\mathrm{F}}$ ) can be evaluated from eq 6 using the Newton method. The calculations were carried out under the conditions that $0<\chi_{12}<2.0,0<\chi_{13}<2.0$ and $0<\chi_{23}<2.0$ with the aid of a Hitac-E600.

\section{RESULTS AND DISCUSSION}

Figure 1 shows the effect of $X_{w}{ }^{0}$ on $v_{1}{ }^{\mathrm{c}}, v_{2}^{\mathrm{c}}$, and $v_{\mathrm{p}}{ }^{\mathrm{c}}$ in the case of $\chi_{12}=0.5, \chi_{13}=0.2$, and $\chi_{23}=1.0$ by full lines. The basic data were reproduced from Figure 5 of ref 12 . The

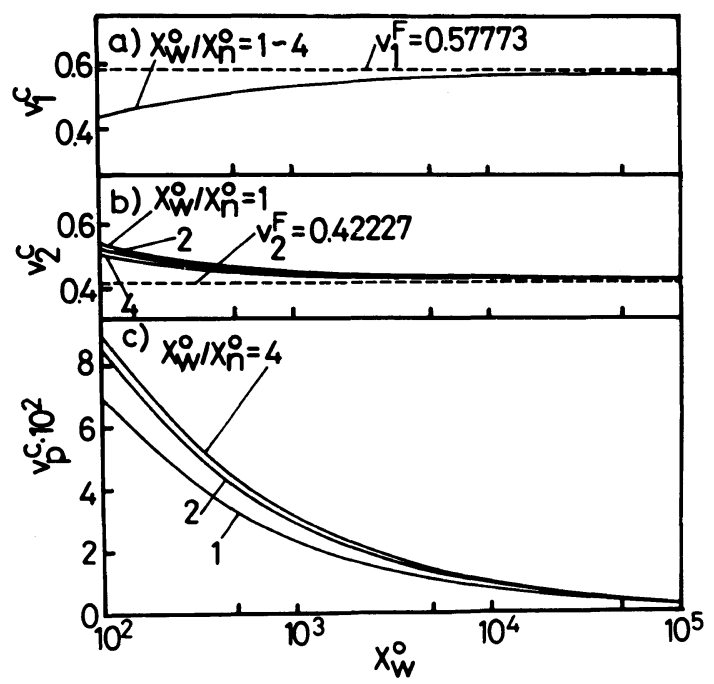

Figure 1. Relations between the critical solution point $\left(v_{1}{ }^{\mathrm{c}}, v_{2}{ }^{\mathrm{c}}\right.$, and $\left.v_{\mathrm{p}}{ }^{\mathrm{c}}\right)$ and $X_{w}{ }^{0}$ for quasi-ternary polymer solutions: Original polymer, Schulz-Zimm type distribution, $X_{w}^{0} / X_{n}^{0}=1,2$, and $4 ; \chi_{12}=0.5, \chi_{13}=0.2$, and $\chi_{23}=1.0$; broken line, Flory solvent compositions.

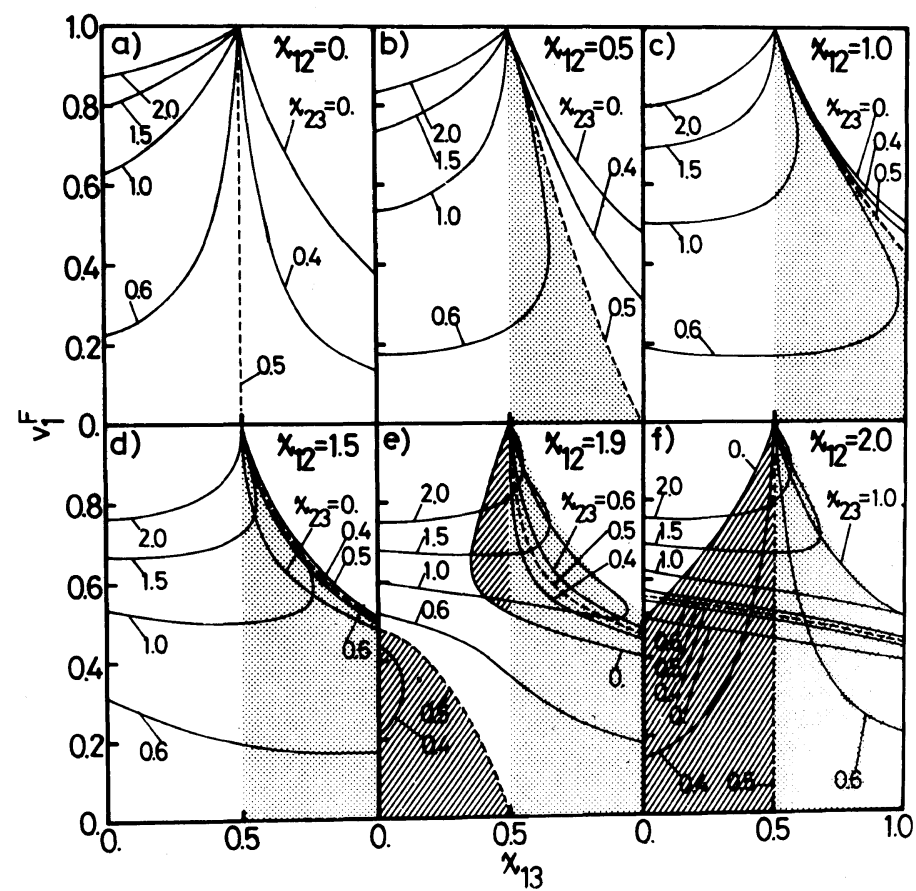

Figure 2. Relations between Flory solvent composition $v_{1}{ }^{\mathrm{F}}\left(=1-v_{2}{ }^{\mathrm{F}}\right)$ and $\chi_{13}$ : a) $\chi_{12}=0$, b) $\chi_{12}=0.5$, c) $\chi_{12}=1.0$, d) $\chi_{12}=1.5$, e) $\chi_{12}=1.9$, f) $\chi_{12}=2.0$; number on curve means $\chi_{23}$. 
broken line in the figure is the Flory solvent composition $\left(v_{1}{ }^{\mathrm{F}}=0.57773\right.$ and $\left.v_{2}{ }^{\mathrm{F}}=0.42227\right)$, calculated from eq 6 . As the theory predicts, with increasing $X_{w}{ }^{0}$, the quantities $v_{1}{ }^{\mathrm{c}}$, and $v_{2}{ }^{\mathrm{c}}$ approach to $v_{1}{ }^{\mathrm{F}}$ and $v_{2}{ }^{\mathrm{F}}$, respectively and $v_{\mathrm{p}}{ }^{\mathrm{c}}$ reduces to zero. This indicates clearly that the mathematical assumption employed in deriving eq 4 is acceptable and eq 6 enables us to calculate $v_{1}{ }^{\mathrm{F}}$ and $v_{2}{ }^{\mathrm{F}}$ with good accuracy.

Figure 2 shows the relations between $v_{1}{ }^{\mathrm{F}}$ and $\chi_{13}$ for fixed values of $\chi_{12}$ and $\chi_{23}$. In the figure the broken line denotes the case of $\chi_{23}=0.5$. For $\chi_{12}=0$ (Figure 2a), the $v_{1}{ }^{\mathrm{F}}$ decreases rapidly with increasing $\chi_{13}$ in the range $\chi_{23}<$ 0.5 , but it increases with increasing $\chi_{13}$ if $\chi_{23}>0.5$. For a specific combination of $\chi_{12}$ and $\chi_{23}\left(\chi_{12}=0\right.$ and $\left.\chi_{23}=0.5\right), \partial v_{1}^{\mathrm{F}} / \partial \chi_{13}$ changes its sign at $\chi_{13}=0.5$. In the case of $\chi_{12}=0$ and $\chi_{13}=\chi_{23}=0.5$, eq 4 (or 6) is satisfied for any combination of $v_{1}$ and $v_{2}$. Note that in a system of $\chi_{12}=0$ and $\chi_{13}=\chi_{23}=0.5$ solvents 1 and 2 are thermodynamically identical and then, the system can be regarded as a quasibinary solution whose $\chi$ is 0.5 . This means that for the system $v_{1}{ }^{\mathrm{F}}$ can be obtained over the whole range of the composition of $v_{1}$. Even in the case of $\chi_{13}>0.5$, there exists the region in which $v_{1}{ }^{\mathrm{F}}$ decreases with an increase in $\chi_{13}$ (for example, $\chi_{12}=0.5$ and $\chi_{23}=0.6$ ). Generally, this region affords us two Flory's compositions for this polymer/solvents system as denoted by shadowed area in the figure. The existence of two Flory's composition for a given polymer/solvent/nonsolvent system has not been unfortunately demonstrated experimentally, but this phenomenon probably is closely correlated with the occurrence of co-solvency. Bamford and Tompa ${ }^{13}$ reported for a cellulose acetate/chloroform/ethyl acetate system the occurrence of co-solvency and two critical solution points. Of course, two critical solution points, corresponding to the infinite polymer, yield two Flory solvent compositions.

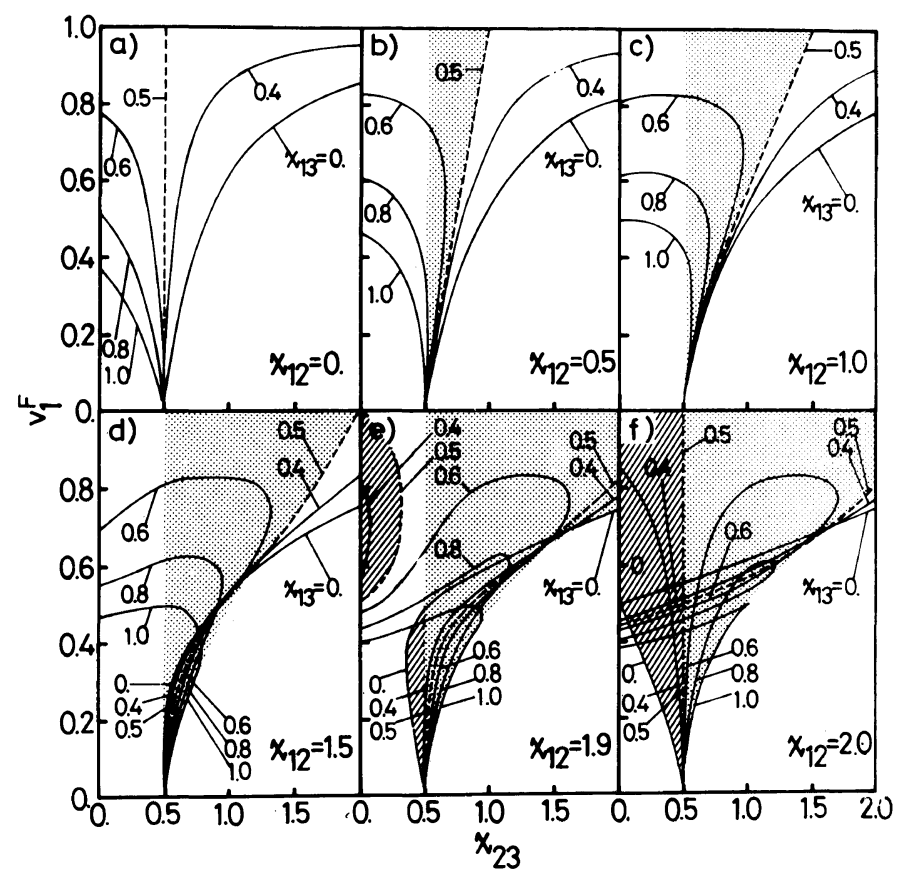

Figure 3. Relations between Flory solvent composition $v_{1}{ }^{\mathrm{F}}\left(=1-v_{2}{ }^{\mathrm{F}}\right)$ and $\chi_{23}$ : a) $\chi_{12}=0$, b) $\chi_{12}=0.5$, c) $\chi_{12}=1.0$, d) $\chi_{12}=1.5$, e) $\chi_{12}=1.9$, f) $\chi_{12}=2.0$; number on curve means $\chi_{13}$. 
As $\chi_{12}$ increases beyond 1.0, the area giving two Flory solvent compositions becomes wide remarkably (Figure 2c-f). For example, in the case of $\chi_{12}=1.0$ and $\chi_{23}=0.6$ (Figure 2c),
$v_{1}^{\mathrm{F}}$ diminishes with increasing $\chi_{13}$ even for $\chi_{13}<0.5$. This means that there is a possibility that as the solubility power of solvent 1 decreases, the composition of solvent 1 (good
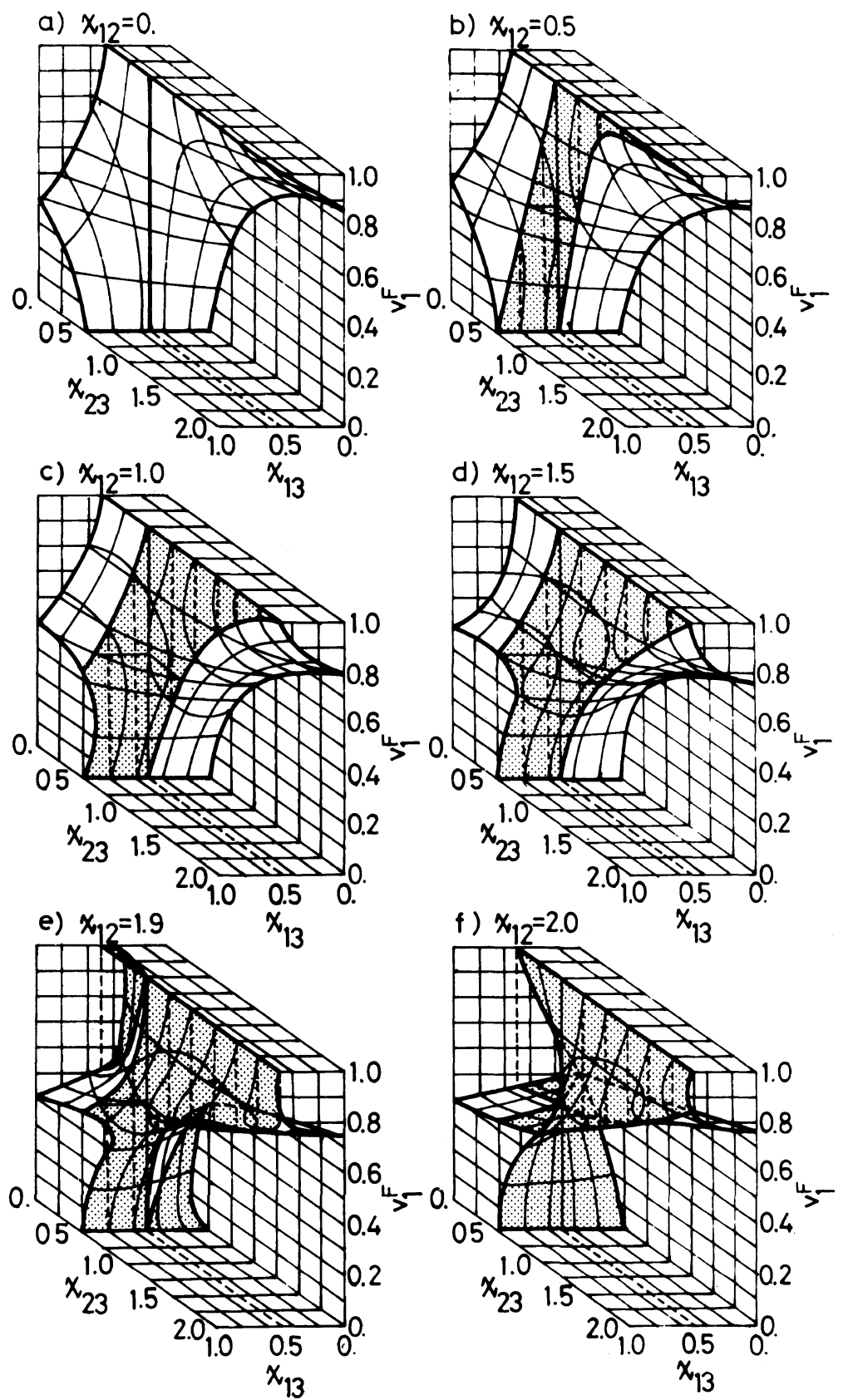

Figure 4. Relations among Flory solvent composition $v_{1}{ }^{\mathrm{F}}\left(=1-v_{2}{ }^{\mathrm{F}}\right), \chi_{13}$ and $\chi_{23}$ : a) $\chi_{12}=0$, b) $\chi_{12}=0.5$, c) $\chi_{12}=1.0$, d) $\chi_{12}=1.5$, e) $\chi_{12}=1.9$, f) $\chi_{12}=2.0$; shadowed area has two Flory solvent compositions. 
solvent) for the Flory solvent decreases unexpectedly. Figure 2e shows that mixing of two good solvents with $\chi_{13}<0.5$ and $\chi_{23}<0.5$ is predicted theoretically to give sometimes a Flory solvent. The area giving the Flory solvent is denoted by hatched area. Inspection of Figure 2 also reveals that in this case, mixing of two good solvents gives two Flory solvent mixtures. This is just reverse of cosolvency phenomena (i.e., co-nonsolvency), but unfortunately it has not yet been confirmed with experiments. In the case of $\chi_{12}=$ 2.0 (Figure 2f), all combinations of $\chi_{13}$ and $\chi_{23}$, except for $\chi_{13}<0.5$ and $\chi_{23}>0.5$ (or $\chi_{13}$ $>0.5$ and $\chi_{23}<0.5$ ), have two Flory solvents compositions.

Figure 3 shows the relations between $v_{1}{ }^{\mathrm{F}}$ and $\chi_{23}$ for the fixed $\chi_{12}$ and $\chi_{13}$ values. The relations were calculated using eq 6 , which is evidently symmetrical with respect to the exchange of solvent 1 and solvent 2 . Figure 3 coincides completely with Figure 2 and no further explanation of Figure 3 is needed.

Figure 4 shows the relations among $v_{1}{ }^{\mathrm{F}}, \chi_{13}$ and $\chi_{23}$, constructed by using the same data as employed in Figures 2 and 3, for fixed $\chi_{12}$ value. Curved surface surrounded with bold full lines corresponds to $v_{1}{ }^{\mathrm{F}}$ for a given combination of three $\chi$ parameters. Reflecting on the symmetry of eq 6 , the axis determined as an interception of two planes; $\chi_{13}=\chi_{23}$ and $v_{1}{ }^{\mathrm{F}}=0.5$ is two-fold axis (not shown in the figure). Any combination of solvent 1 and solvent 2 for a given polymer having three $\chi$ parameters, whose values lie in shadowed area in Figure 4, affords us always two Flory solvents compositions. It is clear that for $\chi_{12}$ $=1.9 \sim 2.0$ two Flory solvents compositions

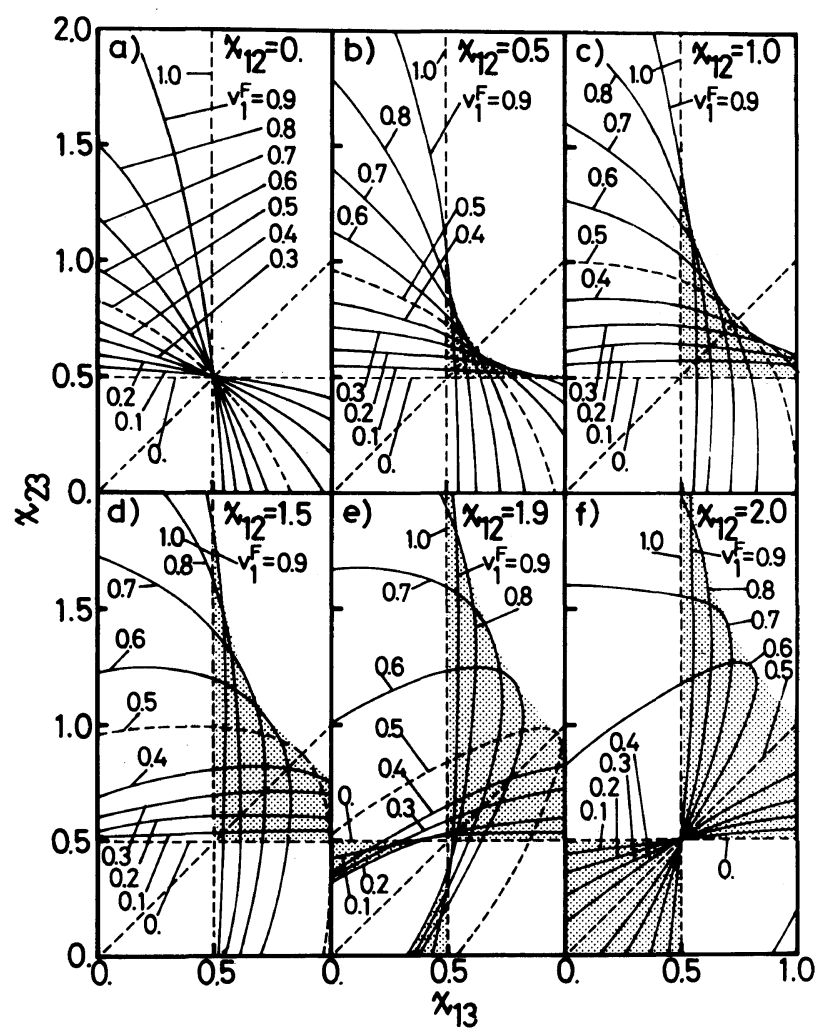

Figure 5. Relations between $\chi_{13}$ and $\chi_{23}$ giving Flory solvent: a) $\chi_{12}=0$, b) $\chi_{12}=0.5$, c) $\chi_{12}=1.0$, d) $\chi_{12}=$ 1.5 , e) $\chi_{12}=1.9$, f) $\chi_{12}=2.0$; shadowed area has two Flory solvent compositions. 
are expected to exist in the range $\chi_{13}<0.5$ and $\chi_{23}<0.5$ : For example, for $\left(\chi_{12}, \chi_{13}, \chi_{23}\right)=$ $(1.9,0,0.4)$ we obtain $v_{1}{ }^{\mathrm{F}}=0.2$ and 0.45 and for $\left(\chi_{12}, \chi_{13}, \chi_{23}\right)=(1.9,0.4,0)$ we obtain $v_{1}{ }^{\mathrm{F}}=$ 0.55 and 0.8 , respectively.

Figure 5 is the relations in figure 4 projected on the $\chi_{13}-\chi_{23}$ plane. Number on the curve means $v_{1}{ }^{\mathrm{F}}$. The relations between $\chi_{23}$ and $\chi_{13}$ for a given $\chi_{12}$ is symmetrical with respect to the plane (vertical mirror), which is perpendicular to the $\chi_{13}-\chi_{23}$ plane and whose interception with the $\chi_{13}-\chi_{23}$ plane gives $\chi_{13}=\chi_{23}$ line. Shadowed boomerang-shape area in the figure is the area, in which two Flory solvent mixtures exist. The $\chi_{13}-\chi_{23}$ relations belonging to the range of $\chi_{13}>0.5$ and $\chi_{23}>0.5$ lie apparently in this area, which expands with an increase in $\chi_{12}$. In addition to this, in the case of $\chi_{12}=1.9$, two wedge shape regions each giving two Flory solvent compositions, are formed even when $\chi_{13}<0.5$ and $\chi_{23}<0.5$, and in the case of $\chi_{12}=2.0$, the total area of $\chi_{13}<0.5$ and $\chi_{23}<0.5$ gives two Flory solvents compositions.

Figure 6 shows the region giving two Flory solvents compositions in $\chi_{12}-\chi_{13}-\chi_{23}$ space. In the figure, the region is shadowed and has a mirror symmetry with respect to the $\chi_{13}=\chi_{23}$ plane, as requested by eq 6 . As well as the

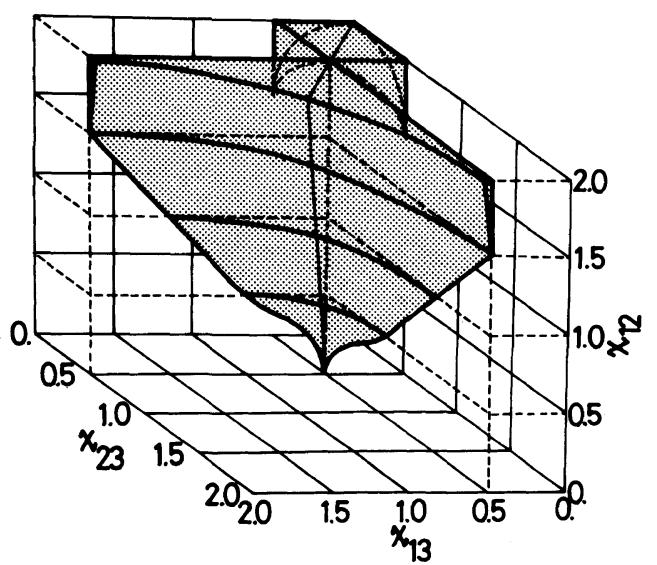

Figure 6. The region giving two Flory solvent compositions in $\chi_{12}-\chi_{13}-\chi_{23}$ space. region of $\chi_{13}>0.5$ and $\chi_{23}>0.5$, relating to cosolvency, the region of $\chi_{13}<0.5$ and $\chi_{23}<0.5$, relating to co-nonsolvency increases its area remarkably with increasing $\chi_{12} \cdot{ }^{14}$

In summary, 1) Flory solvent composition can be theoretically calculated regardless of the polymolecularity of the polymer sample for any polymer-solvent 1-solvent 2 system, if $\chi_{12}, \chi_{13}$, and $\chi_{23}$ and known in advance for the system. 2) Two Flory solvent compositions exist for specific combination of solvents 1 and 2 (i.e., for specific values of $\chi_{12}$, $\chi_{13}$, and $\left.\chi_{23}\right)$. This prediction has not yet been experimentally confirmed. 3) When $\chi_{12}$ $>1.5$ (i.e., when the thermodynamic property of two solvents are greatly different), mixing of two good solvents is theoretically expected to give a Flory solvent. This prediction has also not yet been experimentally ascertained.

\section{REFERENCES}

1. See, for example, J. Bandrup and E. H. Immergut, Ed., "Polymer Handbook," 2nd ed, John Wiley \& Sons, Inc., New York, N. Y., 1974, Chapter IV.

2. P. J. Flory, "Principle of Polymer Chemistry," Cornell Univ. Press, Ithaca, New York, 1953.

3. M. Kurata, "Thermodynamics of Polymer Solution," Harwood Academic Publishers, Chur, London, New York, 1982.

4. H. G. Elias, Makromol. Chem., 33, 140 (1959).

5. H. G. Elias, Makromol. Chem., 50, 1 (1961).

6. D. R. Napper, Polymer, 10, 181 (1969).

7. W. R. Krigbaum and L. H. Sperling, J. Phys. Chem., 64, 99 (1960).

8. K. Kamide and S. Matsuda, Polym. J., 16, 828 (1984).

9. K. Kamide, S. Matsuda, and M. Saito, Polym. J., 17, 1013 (1985).

10. U. Bianchi, V. Magnasco, and C. Rossi, Ric Sci., 28, 1412 (1958).

11. G. Meyerhoff, in J. W. Williams, Ed., "Ultracentrifugal Analysis in Theory and Praxis," Academic Press, New York, N. Y., 1968.

12. K. Kamide and S. Matsuda, Polym. J., 18, 347 (1986).

13. C. H. Bamford and H. Tompa, Trans. Faraday Soc., 46, 310 (1950).

14. S. Matsuda and K. Kamide, Polym. J., 19, 211 (1987). 